\title{
BMJ Open Naoxuekang, Xinnaoshutong and Xuesaitong capsules for treating stroke: a protocol for a randomised controlled trial
}

\author{
Huiling Chen, ${ }^{1,2}$ Hongbo Cao, ${ }^{3}$ Xu Guo, ${ }^{4}$ Meidan Zhao, ${ }^{5}$ Qing Xia, ${ }^{5}$ Bo Chen, ${ }^{5}$ \\ Tieniu Zhao, ${ }^{6}$ Wenyuan Gao ${ }^{1}$
}

To cite: Chen $\mathrm{H}, \mathrm{Cao} \mathrm{H}$, Guo $X$, et al. Naoxuekang, Xinnaoshutong and Xuesaitong capsules for treating stroke: a protocol for a randomised controlled trial. BMJ Open 2017;7:e015983. doi:10.1136/ bmjopen-2017-015983

- Prepublication history for this paper is available online. To view these files, please visit the journal online (http://dx.doi. org/10.1136/bmjopen-2017015983).

Received 16 January 2017 Revised 4 September 2017 Accepted 20 September 2017

\section{CrossMark}

${ }^{1}$ School of Pharmaceutical Science and Technology, Tianjin University, Tianjin, China ${ }^{2}$ School of Chinese Medicine Engineering, Tianjin University of Traditional Chinese Medicine, Tianjin, China

${ }^{3}$ Tianjin Institute of Clinical Evaluation, Tianjin University of Traditional Chinese Medicine, Tianjin, China

${ }^{4}$ School of Language and Culture, Tianjin University of Traditional Chinese Medicine, Tianjin, China

${ }^{5}$ College of Acupuncture and Massage, Tianjin University of Traditional Chinese Medicine, Tianjin, China

${ }^{6}$ School of Chinese Medical, Tianjin University of Traditional Chinese Medicine, Tianjin, China

Correspondence to

Dr Wenyuan Gao;

pharmgao@tju.edu.cn

\section{ABSTRACT}

Introduction After stroke, hemiplegia, dysphasia and facial paralysis can manifest during the convalescent period. Currently, no Chinese patent medicine (CPM) is previously reported to cure each of these symptoms primarily, and thus, there are no relevant instructions for the use of CPM. This study presents a new approach based on comparative effectiveness research to distinguish the curative effects of three CPMs that are often used in stroke convalescence to determine the ideal medicine for the treatment of each symptom.

Methods and analysis In this multicentre and doubleblind clinical trial, stratified randomisation is used to group the patients according to their primary symptoms (hemiplegia, dysphasia and facial paralysis). Three strata will be enrolled, with 80 eligible participants included in each stratum. Each stratum will be randomly and equally divided into four groups, and each group will receive one of the following treatments: Naoxuekang, Xinnaoshutong (XNST), Xuesaitong (XST) or placebo. This study will include two stages: the initial treatment period (30 days) and a follow-up period (180 days). Three replicates for each data point will be completed during this trial. The first visit will occur on day 0 after enrolment, the second visit on day $30 \pm 2$ and the third visit on day $210 \pm 5$. The Delphi technique is adopted to achieve index weighting, which ensures that the evaluation outcome is patient oriented. The weighted index value will be computed as the final measurement index of the outcome.

Ethics and dissemination This study has been approved by the Medical Ethics Committee of Tianjin University of Traditional Chinese Medicine (registration number TJUTCM-EC20160007). The results will be offered for publication in peer-reviewed journals.

Trial registration number This trial was registered with the Chinese Clinical Trial Registry (ChiCTR-IOR-17010397). The date of registration was 11 January 2017.

\section{BACKGROUND}

The overall annual age-standardised incidence and death rate from stroke in the general population of China were 115.61 and 81.88 per 100000 people, respectively, in 1986. ${ }^{1}$ Among these patients, approximately
Strengths and limitations of this study

- A multicentre, prospective and randomised controlled trial design is used.

- The evaluation of patient recovery will include both quality of life and clinical indexes.

- The Delphi technique will be used to assign weight to the scale indexes.

The sample size is small due to funding constraints.

$75 \%$ of them suffered from cerebral ischaemic stroke. Because $70 \%$ to $80 \%$ of the patients who survive stroke experience various degrees of disability and suffer from cognitive handicaps, they require assistance in performing activities of daily life, which severely affects their quality of life and places a heavy burden on their families as well as society. $^{2}$

For the current treatment in the convalescence of stroke, clinical trials have shown that Chinese patent medicine (CPM) has a significant curative effect. ${ }^{3}{ }^{4}$ Many patients in the convalescent or sequelae stage voluntarily take CPMs to treat this disease and prevent recurrence (many CPMs for patients with stroke are over the counter).$^{5}$ There are various kinds of CPMs with similar instructions for the treatment of stroke, such as Naoxuekang (NXK), Xinnaoshutong (XNST) and Xuesaitong (XST) capsules. These three CPMs have been on the market for many years and have received positive clinical feedback. NXK capsules ${ }^{6}$ are manufactured by Shandong HaoFu Pharmaceutical. The main ingredient of NXK is leech, and NXK has been proven to be effective and to cause minor adverse reactions according to clinical observation. ${ }^{7}$ According to the Pharmacopoeia of the People's Republic of China, pregnant women and individuals with bleeding disorders should never be 
treated with NXK. ${ }^{8}$ XNST $^{9}$ capsules are manufactured by Jilin Aodong Taonan Pharmaceutical. The main ingredient of XNST is steroidal saponins of Tribulus terrestris, and XNST has not elicited obvious adverse reactions in clinical trials. ${ }^{10}$ According to the Pharmacopoeia of the People's Republic of China, XNST occasionally leads to mild adverse reactions, such as dry mouth and upset stomach. Patients who have intracranial haemorrhage should not be treated with XNST, while patients with a history of bleeding or blood-low-viscosity-syndrome should be treated with XNST, although with caution. ${ }^{11}$ XST capsules ${ }^{12}$ are manufactured by Kunming Shenghuo Pharmaceutical (Group). The main ingredient of XST is Panax notoginseng saponins, and according to the results of clinical trials, only minor adverse reactions have been reported; thus, it can be given to all patients without restriction. ${ }^{13}$ However, according to the Pharmacopoeia of the People's Republic of China, XST should not be given to pregnant women or people with allergies. ${ }^{14}$ These three CPMs are frequently used for the treatment of stroke, hemiplegia, facial distortion, dysphasia, dark purplish tongue with ecchymosis, cephalophyma or cerebral thrombosis with symptoms caused by encephalorrhagia with high blood pressure. ${ }^{81114}$

In addition, because CPM interprets an illness based on syndrome theories, medical practitioners who are not well educated in Traditional Chinese Medicine
(TCM) theories or those with little clinical experience are often unable to prescribe appropriate CPMs. Therefore, providing instructions on how to use CPMs based on symptoms is more easily understood and accepted by both doctors and patients.

The feasibility of comparative effectiveness research in the evaluation of a CPM

The concept of comparative effectiveness research (CER) was introduced in the 1990s by Mark Boutin, the deputy executive president and chief operating officer of the US National Health Council. ${ }^{15}$ The Agency for Healthcare Research and Quality defined CER as follows: "Comparative effectiveness research is designed to inform healthcare decisions by providing evidence on the effectiveness, benefits, and harms of different treatment options". ${ }^{16}$ The evidence was generated from research studies that compare drugs, medical devices, tests, surgeries or healthcare delivery methods. ${ }^{17}$

In the following years, CER was introduced into the field of clinical research in numerous countries. ${ }^{18}$ In May 2011, CER was introduced into TCM research at the Sixth Annual Meeting of the International Society of Complementary Medicine Research by Claudia M Witt (Institute of Social Medicine, Epidemiology and Health Economics, Charité University Medical Centre, Berlin, Germany). ${ }^{19}$ The outcome of CER focuses on solving the

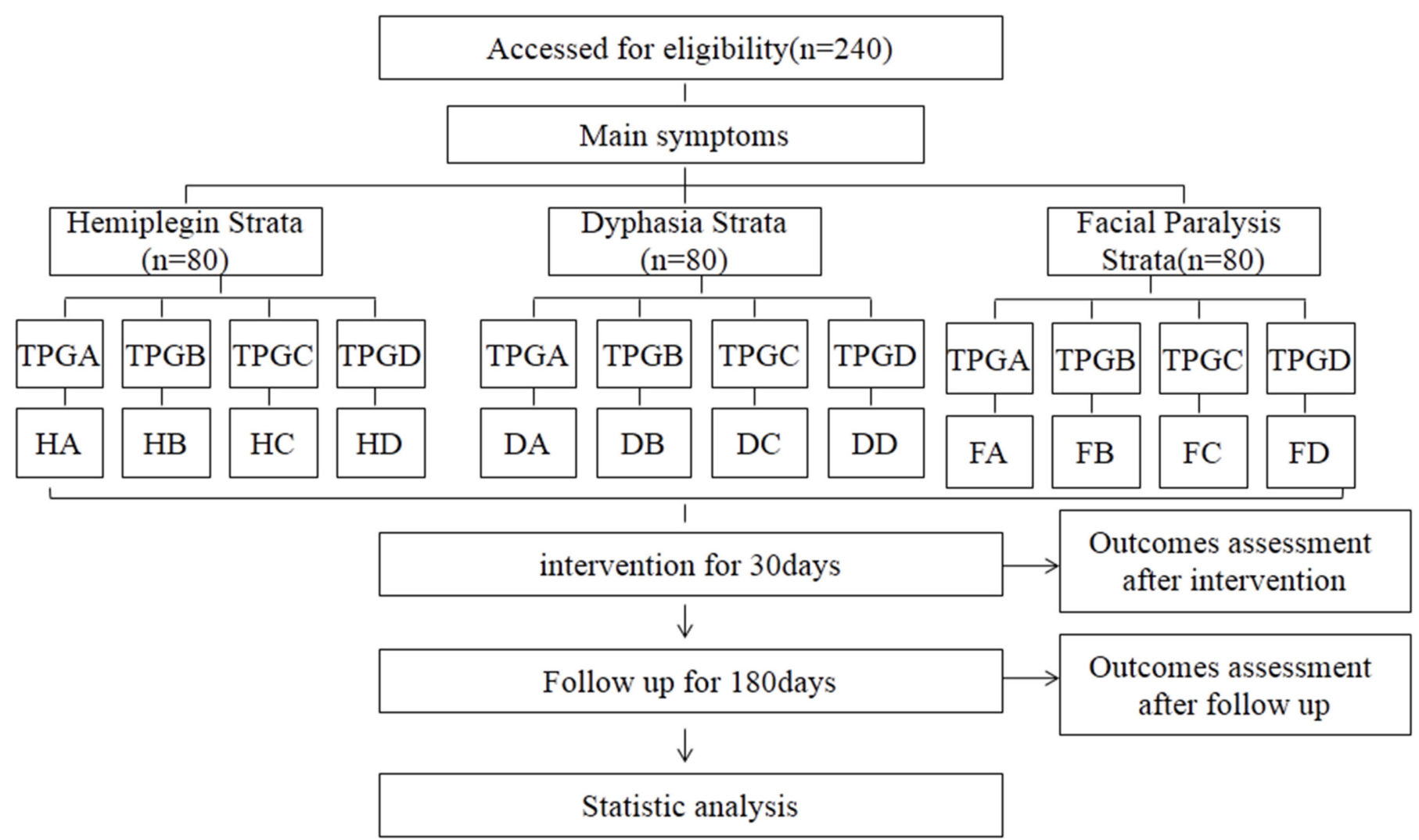

Figure 1 Flow chart of the protocol. DA, dysphasia in group A; DB, dysphasia in group B; DC, dysphasia in group C; DD, dysphasia in group D; FA, facial paralysis in group A; FB, facial paralysis in group B; FC, facial paralysis in group C; FD, facial paralysis in group $\mathrm{D} ; \mathrm{H}$, hemiplegia; TPGA, treatment plan for group A; TPGB, treatment plan for group B; TPGC, treatment plan for group C; TPGD, treatment plan for group D. 
most important problems facing patients. ${ }^{20}$ Thus, CER is concerned with answering questions about the overall effectiveness of interventions rather than efficacy of interventions. ${ }^{21}$ It is more patient oriented, which means it respects the patients' will, focuses on both the quality of life and psychological well-being of patients, aligns with TCM clinical practice.

\section{Effectiveness evaluation based on a patient-oriented theory}

During the convalescent period after stroke, patients suffer from various clinical symptoms and from an inability to perform certain activities of daily living and a lower quality of life. Therefore, evaluation of a patient's recovery should involve both their quality of life, including their mental state, physical condition, psychological condition and social environment and their clinical indexes.

In this study, the WHOQOL-BREF and National Institutes of Health Stroke Scale (NIHSS) are used to assess curative effects and determine their weight with the Delphi technique. A comprehensive score is considered the final curative effect. This approach avoids the randomness of the patient report outcome to some degree, which originates directly from patient reports. ${ }^{22}$

The Delphi technique is used to quantify a qualitative description; thus, it can synthesise the opinions of many experts in a scientific manner to provide reasonable predictions. The Delphi technique asks for, collects and counts individual opinions and judgements by distributing questionnaires to obtain comparatively unanimous opinions on certain issues.

\section{METHODS/DESIGN}

A flowchart of the study protocol is shown in figure 1.

\section{Inclusion criteria}

1. Patients aged 30 to 65 years.

2. Patients experiencing their first stroke event.

3. Diagnosis of unilateral, non-recurring, subacute stroke that is an ischaemic and lacunar type as defined by the International Classification of Diseases 10th Revision through CT or MRI conducted by neurologists.

4. TCM pattern diagnosis of stroke in meridian syndrome.

5. Patients with a score between 6 and 20 for the NIHSS.

6. Duration of 4 to 8 weeks since the original stroke event.

7. Signed informed consent provided by the patient.

The above inclusion criteria will be applied to the experimental group and the control group.

\section{Exclusion criteria}

1. Patients with a known history of allergies or suspected allergies to the medicines used in the study;

2. Patients who suffered from serious heart, liver or kidney-related diseases, blood coagulation dysfunction or severe mental disorders;
3. Patients with other complications;

4. Uncontrolled New York Heart Association class III hypertension (systolic blood pressure $\geq 180 \mathrm{~mm} \mathrm{Hg}$ and/ or diastolic blood pressure $\geq 110 \mathrm{~mm} \mathrm{Hg}$ );

5. Fasting blood glucose $<2.8$ or $>16.8 \mathrm{mmol} / \mathrm{L}$ or with severe complications due to diabetes (eg, diabetic gangrene or peripheral neuropathy);

6. Liver function impairment with values of alanine transaminase or aspartate transaminase over 1.5 fold the upper limit of the normal range;

7. Renal dysfunction with values of serum creatinine over 1.5 fold the upper limit of the normal range;

8. Patients with active peptic ulcers or other haemorrhagic diseases;

9. Patients who are actively participating in other clinical trials or who participated in another clinical trial within the past 90 days.

\section{Treatment plan}

Basic treatment

The intervention programme mainly uses China's Guidelines of Cerebrovascular Disease Prevention and controls as well as the consensus of foreign experts for a reference. This approach considers all the risk factors of apoplexy under strict control. The programme involves the following:

a. An antiplatelet drug: aspirin, taken as prescribed;

b. Blood fat control: simvastatin, taken as prescribed;

c. Blood pressure control: medicines are chosen according to the cause and the severity of high blood pressure. The level of blood pressure is controlled by the researchers;

d. Blood sugar control: according to China's Guidelines of Diabetes Prevention and Control;

e. During the test, all CPMs for activating blood circulation and removing stasis as well as combined Chinese medicine prescriptions will be banned.

\section{The final treatment plans}

The treatment plan for group A: basic treatment +NXKcapsule;

The treatment plan for group B: basic treatment + XNST capsule;

The treatment plan for group C: basic treatment + XST capsule;

The treatment plan for group D: basic treatment + placebo.

The CPM dosage and administration method will follow the doctor's recommendation.

\section{Participant timeline}

An extended observation period will occur to ensure that the curative effect will be accurately determined. This study will include two stages: the initial treatment period (30 days) and a follow-up period (180 days). Three replicates for each data point will be arranged in this trial. The first visit will occur on day 0 after enrolment, the second visit on day $30 \pm 2$ and the third visit on day $210 \pm 5$. 
Table 1 Groups divided according to main symptoms and treatment plans

\begin{tabular}{lllll}
\hline & TPGA & TPGB & TPGC & TPGD \\
\hline H & HA & HB & HC & HD \\
D & DA & DB & DC & DD \\
F & FA & FB & FC & FD \\
\hline
\end{tabular}

$D$, dysphasia; DA, dysphasia in group $A ; D B$, dysphasia in group $\mathrm{B}$; DC, dysphasia in group C; DD, dysphasia in group D; F, facial paralysis; $F A$, facial paralysis in group $A$; $F B$, facial paralysis in group $B$; FC, facial paralysis in group $C ; F D$, facial paralysis in group D; H, hemiplegia; TPGA, treatment plan for group A; TPGB, treatment plan for group B; TPGC, treatment plan for group C; TPGD, treatment plan for group D.

\section{Termination criteria}

Interventions for the trial participants will be discontinued if (1) patients leave the study of their own free will; (2) major errors or serious deviations in study execution (although the plan is good) are identified during the clinical trial protocol that would make it difficult to evaluate the efficacy of the drug; (3) serious adverse events occur during

\begin{tabular}{|c|c|c|c|}
\hline \multirow[b]{3}{*}{ Items } & \multicolumn{3}{|l|}{ Visit } \\
\hline & 1 & 2 & 3 \\
\hline & 0 day & $30 \pm 2$ days & $210 \pm 5$ days \\
\hline \multicolumn{4}{|l|}{ Medical history } \\
\hline $\begin{array}{l}\text { Inclusion/exclusion } \\
\text { criteria }\end{array}$ & $\sqrt{ }$ & & \\
\hline ICF & $\sqrt{ }$ & & \\
\hline $\begin{array}{l}\text { Symptom } \\
\text { differentiation }\end{array}$ & $\sqrt{ }$ & & \\
\hline General information & $\sqrt{ }$ & & \\
\hline $\begin{array}{l}\text { History of medical } \\
\text { treatments and } \\
\text { allergies }\end{array}$ & $\sqrt{ }$ & & \\
\hline Current medications & $\sqrt{ }$ & & \\
\hline Drug distribution & $\sqrt{ }$ & & \\
\hline Drug recovery & & $\sqrt{ }$ & $\sqrt{ }$ \\
\hline Compliance judgement & & $\sqrt{ }$ & $\sqrt{ }$ \\
\hline \multicolumn{4}{|l|}{ Evaluation index } \\
\hline WHOQOL-BREF & $\sqrt{ }$ & $\sqrt{ }$ & $\sqrt{ }$ \\
\hline NIHSS & $\sqrt{ }$ & $\sqrt{ }$ & $\sqrt{ }$ \\
\hline \multicolumn{4}{|l|}{ Safety observation } \\
\hline Vital signs & $\sqrt{ }$ & $\sqrt{ }$ & $\sqrt{ }$ \\
\hline AEs & & $\sqrt{ }$ & $\sqrt{ }$ \\
\hline
\end{tabular}

AEs, adverse events; ICF, informed consent form; NIHSS, National Institutes of Health Stroke Scale the trial and (4) the trial is discontinued by the authorities.

\section{Patient adherence to the instructions}

Patients will receive trial drugs and all necessary healthcare instructions (diet, mental adjustment) for free. Patients are required to maintain appropriate physical activity levels and perform daily exercises. The dosage of the medicine and any remnant shall be recorded in real time, and a drug counting method will be used to monitor the adherence of patients. Patients whose conditions worsen during the trial will be treated appropriately.

\section{Patient grouping}

Patients will be recruited from the First Affiliated Hospital and Second Affiliated Hospital of Tianjin University of TCM, Tianjin Nankai Hospital and Baokang Hospital of TCM in China. Overall, 240 patients who qualify for inclusion will be recruited from all four hospitals during the same time period. To avoid selective bias, the number of patients receiving treatment at each hospital should be balanced and the patients will be divided into three experimental groups and a control group.

1. Patients will be stratified according to their main symptoms. Each stratum will have 80 patients. The patients whose main symptom is hemiplegia will be assigned to stratum $\mathrm{H}$. The patients whose main symptom is dysphasia will be assigned to stratum D. The patients whose main symptom is facial paralysis will be assigned to stratum $\mathrm{F}$.

2. Patients with the same main symptom will be divided into groups A, B, C and D both randomly and equally. Each of the four groups will be treated with different treatment plans, and the curative effects will be recorded. For example, patients whose main symptom is hemiplegia will be treated with plan A in group HA, while patients with the same symptom will be treated with plan B in group HB. Therefore, 12 groups will be generated: group $\mathrm{HA}$, group $\mathrm{HB}$, group $\mathrm{HC}$, group $\mathrm{HD}$, group DA, group DB, group DC, group DD, group FA, group FB, group FC and group FD. Each group will contain 20 patients. Table 1 presents the details according to patient groups.

\section{Effectiveness assessment}

\section{Assessment expert}

One doctor will be assigned to assess the curative effect and record the evaluation results of all volunteers to ensure that observation mode is consistent across the experimental groups and the control group. This doctor will not participate in clinical decisions to avoid evaluator bias. With over 10 years of clinical experience, this physician is an expert in this field and is able provide an accurate assessment of patient symptoms. The WHOQOLBREF and NIHSS questionnaires of all the patients will be recorded by this doctor on day 0 , at the beginning of the experiment; the second visit will occur on day $30 \pm 2$ and the third visit on day $210 \pm 5$. 
Evaluation criteria

\section{Quality of life}

According to the WHOQOL-BREF, quality of life is evaluated by one's mental state, psychological state and physical state, among other factors. ${ }^{23} 24$

\section{Clinical indexes}

The physiological indexes that show the degree of nervous functional defects can be evaluated using the NIHSS.

In this study, the weight table of the indexes will be provided to both the doctors and the patients so that the outcomes can represent the will of each patient and their clinician in a way that aligns with comparative effectiveness research. Each index is designed as a questionnaire with four answers, including 'very important', 'important', 'average/not very important' and 'not important'. Each expert will evaluate the index system according to these four answers.

The formula is as follows:

$$
\text { DWi }=\sum_{j=1}^{m} \text { aij nij/N }
$$

$\mathrm{DW}_{\mathrm{i}-}$ the average value of the importance of the index i $(\mathrm{i}=\mathrm{w}, \mathrm{n})$;

$\mathrm{a}_{\mathrm{ij}}$ - the grade value of the index $\mathrm{i}$;

$\mathrm{j}$ - the grade ordinal;

$\mathrm{N}$ - the number of the experts.

\section{Final curative effects}

Treatment effectiveness is evaluated by comparing the value of each index before treatment with that after treatment. In this study, $\mathrm{W}_{0}$ in equations (eqs) 2 and 3 represents the value of the WHOQOL-BREF before treatment, $W_{1}$ in eqs 2 and 3 indicates the value of the WHOQOL-BREF after treatment and $\mathrm{W}_{2}$ in eq 3 is the value of the WHOQOL-BREF after follow-up. $\mathrm{N}_{0}$ in eqs 2 and 3 represents the value of the NIHSS before treatment, $\mathrm{N}_{1}$ in eqs 2 and 3 indicates the value of the NIHSS after treatment and $\mathrm{N}_{2}$ in eq 3 is the value of the NIHSS after follow-up.

The formula for final curative effects calculation is as follows:

$\mathrm{W}_{1} \times \mathrm{DW}_{\mathrm{W}}+\mathrm{N}_{1} \times \mathrm{DW}_{\mathrm{N}}-\left(\mathrm{W}_{0} \times \mathrm{DW}_{\mathrm{W}}+\mathrm{N}_{0} \times \mathrm{DW}_{\mathrm{N}}\right)(2)$

The formula for curative effects after follow-up is as follows:

$\mathrm{W}_{2} \times \mathrm{DW}_{\mathrm{W}}+\mathrm{N}_{2} \times \mathrm{DW}_{\mathrm{N}}-\left(\mathrm{W}_{0} \times \mathrm{DW}_{\mathrm{W}}+\mathrm{N}_{0} \times \mathrm{DW}_{\mathrm{N}}\right)(3)$

$\mathrm{DW}_{\mathrm{W}}$ in eqs 2 and 3 indicates the weight of the WHOQOL-BREF, and $\mathrm{DW}_{\mathrm{N}}$ in eqs 2 and 3 indicates the weight of the NIHSS.

\section{Data collection methods}

For each patient, measurements will be taken at 0 , $30 \pm 2$ and $210 \pm 5$ days after treatment (table 2 ). The clinical research associates are required to monitor various units on a regular and continuous basis. The data management of the trial follows Good Clinical Data Management Practice. $^{25}$

\section{Sample size}

The sample size in this study is based on the trial results from previous reports and the recommendation of specialists. ${ }^{26-32}$ The values of $\sigma_{i}$ for the WHOQOL-BREF scale of the experimental groups are 12.12, 19.51 and 12.24 , while the value of $\sigma_{i}$ for the WHOQOL-BREF scale of the placebo group is 11.2. The values of $\mu_{i}$ for the WHOQOL-BREF scale of experimental groups are 17.13, 18 and 23.83, while the value of $\mu_{i}$ for the WHOQOLBREF scale of the placebo group is 10.83. According to the calculation, $\mu$ is 17.45 , the type I error is 0.05 and the power is $90 \%$. If the dropout rate is $20 \%$, then 172 patients are needed in the trial (43 patients per group). The values of $\sigma_{i}$ for the NIHSS scale of the experimental groups are 2.6, 7.31 and 3.11 , while the values of $\sigma_{i}$ for the NIHSS scale of the placebo group is 12.5. The values of $\mu_{\mathrm{i}}$ for the NIHSS scale of the experimental groups are 6.85, 4.95 and 6.1 , while the values of $\mu_{i}$ for the NIHSS scale of the placebo group is 1.39 . According to the calculation, $\mu$ is 4.82 , the type I error is 0.05 and the power is $90 \%$. If the dropout rate is $20 \%$, then 232 patients are needed in the trial (58 patients per group).

According to the calculations above and recommendations from the specialists, 240 patients will be used for the trial, with 60 patients per group.

\section{Statistical analysis}

General information about the patients will be registered, including the patient number, sex, age, body mass index, comorbid diseases, type of symptoms, accompanying symptoms, education level and other basic information.

The mean \pm SD deviation will be used for the statistical description of the measurement data. An analysis of variance will be used if the data are normally distributed, while a sum-rank test will be used if the data are not normally distributed or if heterogeneity is found. A comparison among the three experimental groups and the control group will be based on analysis of variance of the repeated measurement data. Considered as a significant difference will be $\mathrm{p}<0.05$.

The evaluation result will be analysed with the method of intention-to-treat analysis (ITT). The ITT analysis method leads to more reliable conclusions, which prevents the cases with a poor effect in the final analysis from being excluded and therefore increases the comparability among the groups. The loss of data will be conducted according to the last observation carried forward principle.

Data analysis will be conducted by researchers who are not involved in experimental or clinical decision-making processes to ensure that bias caused by subjective factors from the researchers is avoided.

\section{Safety}

Adverse events (AEs) and adverse drug reactions (ADRs) will be assessed and reported throughout this study. Furthermore, serious AEs or ADRs appearing during the trial need to be reported to the principal investigator and 
the ethics committee. Every AE will be recorded in detail and closely monitored before stabilisation or resolution.

HuilingChen will cooperate with the physician in charge to evaluate the severity and determine the cause of the events. All relevant AEs will be reported to the Institutional Review Board of the First Affiliated Hospital of Tianjin University of TCM within the relevant time frames. HLC will be responsible for reporting all AEs. The coordinators will be responsible for establishing standard procedures and for training the relevant staff before trial initiation. Regular monitoring will be used to ensure that all AEs are identified and addressed appropriately.

The incidence of AEs and ADRs is compared among various groups using the $\chi^{2}$ test with the level of significance set at $\mathrm{p}<0.05$.

Auditors are required to audit trials by checking documents at the midpoint and endpoint of the study, and the process will occur independently of the investigators and the study sponsor.

\section{Randomisation, blinding and allocation concealment}

Patients will be assigned randomly by the stratified randomisation method (1:1:1:1); the stratification factor is the main symptom (hemiplegia, dysphasia and facial paralysis). Randomisation of the patients will be completed using an independent data centre with an interactive voice response system, and a random number will be generated by this data centre for each patient. Original copies of the blind codes will be sealed in a light-proof envelope; one copy will be retained by the major research unit, and another copy will be kept by the applicant of the trial. The envelopes will not be opened prior to formal statistical analysis. If a patient is eligible, that patient number will be allocated by the doctors. These patient numbers are merely serial numbers for labelling patients. The test medicine will be coded first, and then it will be placed in indistinguishable containers by specially assigned personnel who will not participate in this trial. In addition, medicine assignments will be in opaque envelopes and kept confidential by the trial management board. The original capsule shells of NXK, XNST and XST will be exchanged for new uniform capsule shells provided by the pharmaceutical factory of Tianjin University of TCM. The placebo amylum will be placed into capsule shells that are identical to the shells of the experimental drugs. Therefore, the volunteers, doctors, participating nurses, trial coordinators, statisticians and outcome assessors will be blinded to the treatment assignments.

If emergencies occur or treatments are needed, the person responsible for the participating units will immediately report to CRA and the major investigators, and patient unblinding will be performed only with their approval. Once the allocation is unblinded, the investigators must comply with the trial requirements when analysing and recording data.

Acknowledgements We gratefully acknowledge support from the National Natural Science Foundation of China (No.81202849, No. 30600834, and No. 81603659). We are especially grateful to all the trial staff working at our research affiliates. We also thank Tie YU for his revision of the draft.

Contributors HC designed the protocol and wrote the draft. WG conceived the study, and HC revised the manuscript critically for important intellectual content. XG and MZ edited the manuscript and contributed to the final draft. TZ was responsible for all statistical analysis in this trial. QX and BC helped conduct the trial. All authors have carefully read and approved the final manuscript. The trial sponsor designed this protocol, prepared the draft and was responsible for the selection of research units, researchers and drug resources. The costs, such as those for publishing the article and purchasing CPMs are supported by the funders.

Funding The study is funded by the National Natural Science Foundation of China (No.81202849, No. 30600834 and No. 81603659).

Competing interests None declared.

Patient consent Obtained.

Ethics approval This study has been approved by the Medical Ethics Committee of Tianjin University of Traditional Chinese Medicine (registration number TJUTCM-EC20160007). If the protocol needs to be modified, we will reapply for ethical approval. All patients are required to sign an informed consent prior to participation, and the researcher is required to explain the procedures and the objectives of the research, including details regarding the methods to be used, the risks and benefits and the possibility of inclusion in a control or experimental group. The study follows the principle that all information related to patients is confidential; their names will not appear in any records.

Provenance and peer review Not commissioned; externally peer reviewed.

Data sharing statement The results of this pilot study will be disseminated via peer-reviewed publications and conference presentations. All the data will be available upon request.

Open Access This is an Open Access article distributed in accordance with the Creative Commons Attribution Non Commercial (CC BY-NC 4.0) license, which permits others to distribute, remix, adapt, build upon this work non-commercially, and license their derivative works on different terms, provided the original work is properly cited and the use is non-commercial. See: http://creativecommons.org/ licenses/by-nc/4.0/

(c) Article author(s) (or their employer(s) unless otherwise stated in the text of the article) 2017. All rights reserved. No commercial use is permitted unless otherwise expressly granted.

\section{REFERENCES}

1. He J, Klag MJ, Wu Z, et al. Stroke in the people's Republic of China I. Geographic variations in incidence and risk factors. Stroke 1995;26:2222-7.

2. Yang HY. Analyze on the application principle of Chinese patent medicine and the situation of irrational drug use [in Chinese]. Clinical drug therapy 2009;7:36-8.

3. Yang HF. Evaluation the clinical efficacy and safety of traditional Chinese medicine which promoting blood circulation, removing blood stasis, supplementing qi and dredging collaterals combined with western medicine in the treatment of ischemic stroke [in Chinese]. Practical journal of cardiac cerebral pneumal and vascular disease 2012;20:462-3.

4. ZX W. Curative effect observation of Xianfanghuoming decoction combined with western medicine in the treatment of qi stagnation and phlegm type of stroke [in Chinese]. Chinese health nutrition 2010;12:164-5.

5. Rao LX. Analysis of the application of Chinese and western medicine in the treatment of stroke rehabilitation [in Chinese]. Journal of Traditional Chinese Medicine 2010;5:1873-4.

6. Wang JC, Duan YJ, Wg A. Curative effect observation of 80 cases of acute cerebral infarction treated with NaoxueKang capsule [in Chinese]. Shanxi Med Journal 2004;17:2008-9.

7. Gao WH, Tian MY. Effects of Naoxuekang capsules on hemorrheology of cerebral infarction patients [in Chinese]. $\mathrm{HeBei}$ Medicine 2007;8:940-2.

8. Chinese Pharmacopoeia Commission. Pharmacopoeia of the People's Republic of China. Chinese medicine science and technology press, 2015:111.

9. Qu J. Summary of clinical application of cardiac and Xinnaoshutong Capsule [in Chinese]. China Health Industry 2011;7:60.

10. Lin $\mathrm{X}, \mathrm{HI} \mathrm{Y}$, Lin $\mathrm{HJ}$, et al. The clinical treatment effective analysis of cerebral infarction sequelae of Xinnaoshutong capsule. Chinese 
Journal of Intergartive Medicine on Cardio/Cerebrovasascular Disease 2012;2:167-8.

11. Chinese Pharmacopoeia Commission. Pharmacopoeia of the People's Republic of China. Chinese medicine science and technology press 2015;16:26.

12. Sy $L$, Zhang $S N, Z s L$. To observe the clinical curative effect of Xuesaitong soft capsule in the treatment of stroke [in Chinese]. Hebei Medical Journal 2010;5:1058-9.

13. SY L, Li Min LWH, Shi QY. Efficacy observation of Xuesaitong soft capsule on the improvement of life self-care ability in patients with cerebral infarction. China Pharmacy 2012;48:4552-3.

14. Chinese Pharmacopoeia Commission. Pharmacopoeia of the People's Republic of China. : Chinese medicine science and technology press, 2015:17: 108.

15. Anon. Comparative effectiveness: its origin, evolution, and influence on health care. J Oncol Pract 2009;5:80-2.

16. National Information Center on Health Services Research and Health CareTechnology (NICHSR). Comparative Effectiveness Research (CER). U.S: National Library of Medicine, 2014. http://www.nlm.nih. gov/hsrinfo/cer.html. (accessed 7 Mar 2014).

17. Fung V, Brand RJ, Newhouse JP, et al. Using medicare data for comparative effectiveness research: opportunities and challenges. Am J Manag Care 2011;17:489-96.

18. Patel I, Rarus R, Tan X, et al. Investigation of comparative effectiveness research in Asia, Europe, and North America. Indian $J$ Pharmacol 2015;47:85-93.

19. National Information Center on Health Services Research and Health CareTechnology (NICHSR). National Institutes of Health: Comparative Effectiveness Research (CER). Bethesda: National Library of Medicine, 2014. http://www.nlm.nih.gov/hsrinfo/cer.html. (accessed 7 Mar 2014).

20. Moore CL, Broder J, Gunn ML, et al. Comparative effectiveness research: alternatives to "Traditional" computed tomography use in the acute care setting. Acad Emerg Med 2015;22:1465-73.

21. Bekhuis T, Demner-Fushman D, Crowley RS. Comparative effectiveness research designs: an analysis of terms and coverage in Medical Subject Headings (MeSH) and Emtree. J Med Libr Assoc 2013;101:92-100.

22. Valdes K, MacDermid J, Algar L, et al. Hand therapist use of patient report outcome (PRO) in practice: a survey study. $J$ Hand Ther 2014;27:299-308.

23. Yao G, Chung CW, Yu CF, et al. Development and verification of validity and reliability of the WHOQOL-BREF Taiwan version. $J$ Formos Med Assoc 2002;101:342-51.

24. Liang WM, Chang $\mathrm{CH}$, Yeh YC, et al. Psychometric evaluation of the WHOQOL-BREF in community-dwelling older people in Taiwan using Rasch analysis. Qual Life Res 2009;18:605-18.

25. Society for clinical data management. Good Clinical Data Management practice guidance document [Online]. $2007 \mathrm{http}: / / \mathrm{www}$. scdm.org/sitecore/content/be-bruga/scdm/Publications/gcdmp. aspx/

26. $\mathrm{Jb} \mathrm{L}, \mathrm{Cm} \mathrm{N}$, Rui $\mathrm{H}$, et al. Influencing factors of the quality of life in patients with acute stroke. Chin J Phys Med Rehabjl 2005;9:542-4.

27. Jm F, Xd G, Yao YH, et al. Effect in quality of life of scalp acupuncture for long time combined with rehabilitation training on the stroke patients with cognitive disfunction. Chinese Archives of Traditional Chinese Medicine 2010;4:796-8.

28. Qiu XY. Effect of health education on the quality of life of stroke patients. Asia-Pacific Traditional Medicine 2008:9:101-2.

29. Meng T, Gang W. Treatment Effective of ischemic stroke for limb dysfunction by internal and external wiping combined with pricking blood and cupping therapy with Tongfu Huatan Decoction. Modern Journal of Integrated Traditional Chinese and Western Medicine 2016;25:2014-6.

30. Zhao GZ, Ying Z. Clinical study on treatment of acute cerebral hemorrhage with NaoXueKang. Chin J Crit Care Med 1995;5:38-40.

31. Wang MH W. Effect of Xuesaitong soft capsule on acute lacunar cerebral infarction combined with cerebral microbleeds. Chinese Journal of Clinical Pharmacology and Therapeutics 2017;22:574-9.

32. Huang HQ. Clinical observation on treatment of acute cerebral infarction with XinnaoShuTong. Journal of Changchun University of Traditional Chinese Medicine 2010;12:866. 